\title{
Sobre la síntesis óptima de mecanismos
}

\section{On the optimal synthesis of mechanisms}

FOSTER-VÁZQUEZ, Ixshel Jhoselyn ${ }^{1 *}$, PORTILLO-VÉLEZ, Rogelio de Jesús ${ }^{2}$ y VAZQUEZSANTACRUZ, Eduardo ${ }^{1}$

\author{
${ }^{1}$ Universidad Autónoma del Carmen \\ ${ }^{2}$ Universidad Veracruzana
}

ID $1^{\mathrm{er}}$ Autor: Ixshel Jhoselyn, Foster-Vázquez / ORC ID: 0000-0002-0735-9003, CVU CONACYT ID: 850522

ID $1^{\text {er }}$ Coautor: Rogelio De Jesús, Portillo-Vélez / ORC ID: 0000-0001-8290-617X, CVU CONACYT ID: 205463

ID $2^{\text {do }}$ Coautor: Eduardo, Vazquez-Santacruz / ORC ID: 0000-0002-7493-4450, CVU CONACYT ID: 172895

DOI: $10.35429 /$ JSL.2019.19.6.16.22

Recibido 23 Abril, 2019; Aceptado 30 Junio, 2019

\section{Resumen}

En el proceso de diseño en ingeniería, es de particular relevancia el planteamiento de los problemas que han de ser resueltos para garantizar un diseño óptimo de las propuestas de solución. No existe una regla general para esto, y en el caso particular de la síntesis de mecanismos planos, la solución depende estrechamente del planteamiento del problema de diseño o síntesis del mecanismo. El objetivo de este trabajo es presentar una propuesta al planteamiento del problema de síntesis de un mecanismo plano de cuatro barras para seguimiento de trayectorias cartesianas. El problema de síntesis del mecanismo se plantea como un problema de optimización no lineal con restricciones no lineales. En este trabajo se consideran cuatro diferentes enfoques con el fin de mostrar el impacto del planteamiento inicial del problema de optimización en su solución. La solución de los cuatro problemas de optimización se obtiene mediante cálculos numéricos con algoritmos genéticos. Los resultados numéricos de los cuatro planteamientos se comparan bajo condiciones justas y muestran la gran influencia del planteamiento inicial del problema en su solución.

Mecanismos, Optimización, Planteamiento del problema

\begin{abstract}
In the engineering design process, it is of particular relevance the problem statement that has to be solved to guarantee an optimal design. There is no general rule for this, and in the particular case of the synthesis of flat mechanisms, the solution strongly depends on the problem statement for the design or mechanism synthesis. The object this paper is presenting one proposal at synthesis problem of a four-bar flat mechanism for cartesian trajectory tracking. The mechanism synthesis problem is stated as a nonlinear optimization problem with non linear constraints. Four different approaches are considered in order to demonstrate the impact of the considered statement of the optimization problem for its solution. The solution of the four optimization problems is obtained by means of numerical calculations using genetic algorithms. The numerical results of the four optimization problem statemens are compared under fair circumstances and they depict the great influence of the initial problem statement for its solution.
\end{abstract}

Mechanisms, Optimization, Problem statement

Citación: FOSTER-VÁZQUEZ, Ixshel Jhoselyn, PORTILLO-VÉLEZ, Rogelio de Jesús y VAZQUEZ-SANTACRUZ, Eduardo. Sobre la síntesis óptima de mecanismos. Revista de Simulación y Laboratorio. 2019, 6-19: 16-22

\footnotetext{
*Correspondencia al Autor (Correo electrónico: iixshell@gmail.com)

$\dagger$ Investigador contribuyendo como primer Autor
} 


\section{Introducción}

El planteamiento del problema en el proceso de diseño en ingeniería es una tarea amplia y sumamente importante que necesita de diversas habilidades y conocimientos para obtener resultados satisfactorios y un diseño óptimo. En el caso particular de diseño de mecanismos y en específico de la síntesis de mecanismos planos la solución recae en el planteamiento del problema de diseño o síntesis del mecanismo y en este trabajo, se presenta el planteamiento del problema de síntesis dimensional de un mecanismo plano de cuatro barras para el seguimiento de trayectorias cartesianas.

El problema de síntesis de mecanismos es un tema muy estudiado en la comunidad científica y estos se pueden clasificar en las técnicas utilizadas para resolverlos. Se tienen técnicas modernas como la optimización no lineal como en [1], en donde Vallejo, et al, proponen un método para la síntesis óptima de mecanismos planos con pares inferiores y la configuración de cualquier tipo; Vasiliu, et al, resolvieron un problema de síntesis dimensional de mecanismos planares utilizando redes neuronales, propusieron un método original para sintetizar las dimensiones de un mecanismo plano [2].

También se tienen trabajos utilizando algoritmos genéticos como en [3], en donde Quintero, et al, realizaron una síntesis de generación de trayectoria y movimiento para múltiples posiciones, haciendo una extensión de un mecanismo de cuatro barras, a uno de seis barras para la generación de guiado de cuerpo. Cabrera, et al, realizaron un método para la síntesis óptima de mecanismos trabajando con algoritmos genéticos, utilizaron los problemas de síntesis de mecanismos planos de cuatro barras para probar el método [4]. Eduardo, et al, presentaron la síntesis de un mecanismo de cuatro barras para el seguimiento de una trayectoria lineal de seis puntos, presentó el problema de síntesis como un problema de optimización numérica, utilizó evolución diferencial como parte de la técnica de optimización [5].

Así como estos trabajos existen aún más donde cada uno presenta un enfoque diferente en el planteamiento del problema para la resolución de problemas de síntesis de mecanismos.
En este trabajo se presenta en la sección 2 una explicación en general de lo que es síntesis, se introduce al problema de síntesis del mecanismo de cuatro barras (M4B), se obtiene la cinemática del M4B y la del acoplador, por último, se define la trayectoria deseada. En la sección 3 se ofrece una breve explicación de un algoritmo genético y de las partes que lo conforman. En la sección 4 se plantea el problema de síntesis como un problema de optimización numérica (estrategia de optimización). En la sección 5 se describe la metodología utilizada en este trabajo, y por último en la sección 6 se presentan los resultados, en la sección 7 las conclusiones del trabajo y en la sección 8 se tienen las referencias.

\section{Síntesis de mecanismos}

En mecánica el termino síntesis, se refiere al diseño o creación de un mecanismo para obtener un movimiento deseado [11].

La síntesis o el problema de síntesis se divide en tres partes, síntesis de tipo, síntesis de número y síntesis dimensional.

La síntesis de tipo se refiere a la clase de mecanismo seleccionado, por ejemplo, un sistema de levas, bandas, poleas o mecanismos planares [6].

La síntesis de número se ocupa del número de eslabones y de articulaciones o pares que se requieren para obtener una movilidad determinada [6].

La síntesis dimensional es la encargada de determinar las dimensiones de los eslabones de forma individual [6].

El problema de síntesis dimensional se subdivide en 3 categorías: generación de funciones, guiado de sólidos y generación de trayectoria.

En la categoría de generación de funciones, se trata de que un elemento de salida gire, oscile, o tenga un movimiento alternativo, según una función del tiempo, o bien, una función del movimiento de entrada especificada [6].

La categoría de guiado de solidos reside en mover un objeto de una posición a otra [6]. 
Por último, en la categoría de generación de trayectoria implica lograr que un punto del acoplador genere una trayectoria previamente establecida [6].

\section{Problema de síntesis del mecanismo de cuatro barras}

Para el problema de síntesis de mecanismo, se presenta el mecanismo de cuatro barras (M4B) en la figura 1, las partes que lo componen son: $\bar{r}_{1}$ eslabón de referencia y su ángulo $\theta_{1}, \bar{r}_{2}$ eslabón de entrada o manivela y su ángulo $\theta_{2}, \bar{r}_{3}$ eslabón acoplador y su ángulo $\theta_{3}, \bar{r}_{4}$ eslabón de salida u oscilador y su ángulo $\theta_{4}, \bar{r}_{p}$ eslabón guía y su ángulo $\alpha$.

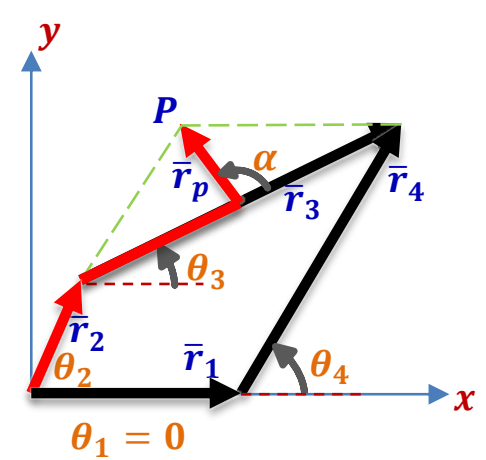

Figura 1 Mecanismo de cuatro barras

El primer paso para el planteamiento de un problema de síntesis es identificar qué tipo de síntesis y qué tarea realizará el mecanismo que se desea diseñar, en el presente trabajo se plantea un problema de síntesis dimensional de un mecanismo plano de cuatro barras para el seguimiento de una trayectoria.

\section{Cinemática del M4B}

Una vez establecido el mecanismo, el tipo de síntesis y la tarea a realizar, se debe obtener la cinemática del mecanismo. La cinemática es el estudio de la posición, el desplazamiento, la rotación, la rapidez, la velocidad y la aceleración [6] y para este trabajo sólo se considera el análisis de posición del mecanismo.

Para el cálculo de la cinemática del M4B primero se obtiene la ecuación de circuito cerrado descrita por la ecuación 1 (Para una explicación detallada consultar en [7].

$r_{2}+r_{3}=r_{1}+r_{4}$
Aplicado la forma polar a cada uno de los elementos de la ecuación 1 , se obtiene:

$r_{2} e^{J \theta_{2}}+r_{3} e^{j \theta_{3}}=r_{1} e^{j \theta_{1}}+r_{4} e^{j \theta_{4}}$

Aplicando la ecuación de Euler a la ecuación 2 y separando la parte real de la imaginaria se obtiene la ecuación 3.

$r_{2} \cos \theta_{2}+r_{3} \cos \theta_{3}=r_{1} \cos \theta_{1}+r_{4} \cos \theta_{4}$ $r_{2} \sin \theta_{2}+r_{3} \sin \theta_{3}=r_{1} \sin \theta_{1}+r_{4} \sin \theta_{4}$

Para obtener la posición angular de $\theta_{4}$, el lado izquierdo del sistema de ecuaciones (3) debe expresarse en términos de $\theta_{3}$, como la ecuación 4.

$r_{3} \cos \theta_{3}=r_{1} \cos \theta_{1}-r_{2} \cos \theta_{2}+r_{4} \cos \theta_{4}$ $r_{3} \sin \theta_{3}=r_{1} \sin \theta_{1}-r_{2} \sin \theta_{2}+r_{4} \sin \theta_{4}$

Elevando al cuadrado y sumando sus términos de la ecuación 4, se obtiene la ecuación de Freudenstein en forma compacta [6], ecuación 5.

$A \cos \theta_{4}+B \sin \theta_{4}+C=0$

donde:

$A=2 r_{4}\left(r_{1} \cos \theta_{1}-r_{2} \cos \theta_{2}\right)$
$B=2 r_{4}\left(r_{1} \sin \theta_{1}-r_{2} \sin \theta_{2}\right)$
$C=r_{1}^{2}+r_{2}^{2}-r_{3}^{2}+r_{4}^{2}-2 r_{1} r_{2} \cos \left(\theta_{1}+\theta_{2}\right)$

La ecuación 5 presenta una única incógnita $\theta_{4}$ pero es argumento de dos funciones diferentes, por lo tanto, se deben aplicar las siguientes identidades trigonométricas (ecuación 6).

$\cos \theta=\frac{1-\tan ^{2} \frac{\theta}{2}}{1+\tan ^{2} \frac{\theta}{2}}$
$\sin \theta=\frac{2 \tan \frac{\theta}{2}}{1+\tan ^{2} \frac{\theta}{2}}$

Sustituyendo la ecuación 6 en la ecuación 5 , se obtiene la ecuación lineal de segundo orden (ecuación 7).

$(C-A) \tan ^{2} \frac{\theta_{4}}{2}+2 B \tan \frac{\theta_{4}}{2}+(A+C)=0$

Resolviendo la ecuación 7, se obtiene la posición angular de $\theta_{4}$ (ecuación 8).

$\theta_{4}=2 \tan ^{-1}\left(\frac{-B \pm \sqrt{A^{2}+B^{2}-C^{2}}}{C-A}\right)$

FOSTER-VÁZQUEZ, Ixshel Jhoselyn, PORTILLO-VÉLEZ, Rogelio de Jesús y VAZQUEZ-SANTACRUZ, Eduardo. Sobre la síntesis óptima de mecanismos. Revista de Simulación y Laboratorio. 2019. 
Para obtener $\theta_{3}$ se debe de realizar un procedimiento similar al anterior, por lo tanto, de la ecuación 3 se obtiene la ecuación de Freudenstein en forma compacta (ecuación 9) [6].

$$
D \cos \theta_{3}+E \sin \theta_{3}+F=0
$$

donde:

$$
\begin{aligned}
& D=2 r_{3}\left(r_{2} \cos \theta_{2}-r_{1} \cos \theta_{1}\right) \\
& E=2 r_{3}\left(r_{2} \sin \theta_{2}-r_{1} \sin \theta_{1}\right) \\
& F=r_{1}^{2}+r_{2}^{2}+r_{3}^{2}-r_{4}^{2}-2 r_{1} r_{2} \cos \left(\theta_{2}-\theta_{1}\right)
\end{aligned}
$$

Por lo tanto, $\theta_{3}$ está dado por la ecuación 10.

$$
\theta_{3}=2 \tan ^{-1}\left(\frac{-E \pm \sqrt{D^{2}+E^{2}-F^{2}}}{F-D}\right)
$$

Para definir la configuración del M4B, se debe elegir el signo apropiado del radical de las ecuaciones 8 y 10 . En la tabla 1 se muestra los signos que deben adoptar los radicales de acuerdo con la configuración del mecanismo que se desee.

\begin{tabular}{|l|c|c|}
\hline Configuración del M4B & $\theta_{3}$ & $\theta_{4}$ \\
\hline Abierta & $+\sqrt{ }$ & $-\sqrt{ }$ \\
\hline Cruzada & $-\sqrt{ }$ & $+\sqrt{ }$ \\
\hline
\end{tabular}

Tabla 1 Selección del signo del radical de acuerdo con la configuración deseada del mecanismo

\section{Cinemática del acoplador}

Para el seguimiento de la trayectoria en el M4B, el punto de interés $P$ se encuentra situado en el punto medio del acoplador, su posición está descrito por la ecuación 11.

$P_{x}=r_{2} \cos \theta_{2}+\Delta r_{3} \cos \theta_{3}+r_{p} \cos \alpha$

$P_{y}=r_{2} \sin \theta_{2}+\Delta r_{3} \sin \theta_{3}+r_{p} \sin \alpha$

\section{Trayectoria deseada}

La trayectoria deseada que se seleccionó para el M4B está definida en la ecuación 12.

$X_{D}=\mathrm{a} \cos \alpha+b$

$Y_{D}=c \sin \alpha+d$

La trayectoria descrita en la ecuación 12 es una trayectoria elíptica con centro en $(b, d)$, donde $a$ define el ancho de la elipse y $c$ su altura.

\section{Algoritmos genéticos}

Los algoritmos genéticos (AG) son métodos estocásticos que pueden usarse para resolver problemas de búsqueda y optimización, están basados en la teoría de la selección natural y fueron desarrollados por John H. Holland a principios de 1960 [8]. Un esquema reducido del funcionamiento de un $\mathrm{AG}$ es el siguiente:

1. El AG comienza creando una población inicial aleatoria

2. Calcula la aptitud de cada individuo

3. Hace una selección probabilística del individuo en base a la aptitud.

4. Aplica operadores genéticos para crear la siguiente población.

5. Se repiten los puntos 2 al 4 hasta que el AG satisfaga cierta condición.

En la figura 2 se presenta un diagrama de flujo del esquema reducido del funcionamiento de los AG.

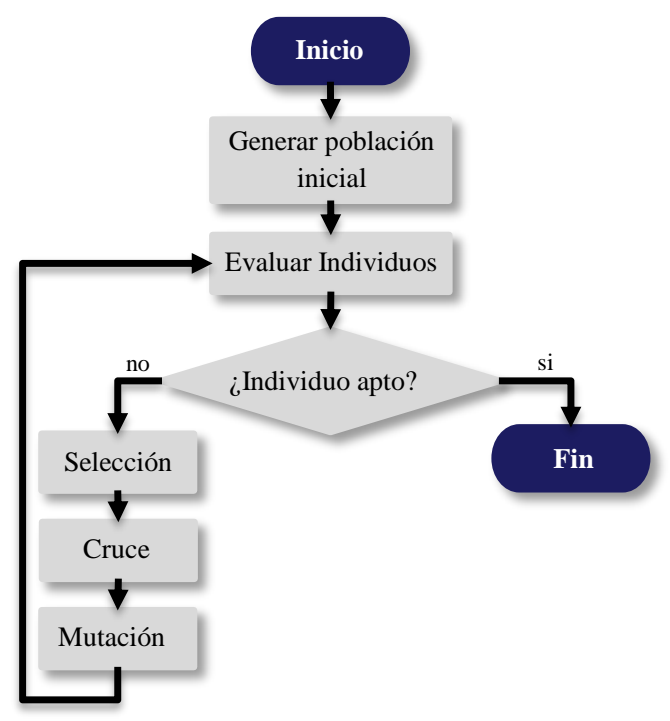

Figura 2 Diagrama de flujo del funcionamiento de un AG

El algoritmo genético está conformado por un conjunto $\mathrm{P}$ (población) de $\mathrm{N}$ individuos, esta población es evaluada por una serie de operadores descritos seguidamente.

Los operadores básicos de un AG son:

- Selección: La función de selección elige a los individuos (padres) para formar la próxima generación en función del valor aptitud.

- Cruce: La cruza ocurre cuando los individuos padres intercambian genes para crear un nuevo individuo (hijo). 
Mutación: La mutación se realiza después de la cruza y es la variación de los genes del individuo de forma aleatoria.

\section{Estrategia de optimización}

El objetivo de este trabajo es el planteamiento del problema de síntesis dimensional de un M4B para el seguimiento de trayectorias cartesianas, en éste se considera obtener las dimensiones del M4B para cumplir con una trayectoria previamente establecida en el punto $\left(P_{x}, P_{y}\right)$ y en la sección 2 se estableció la cinemática del M4B y la cinemática del acoplador, también se describió previamente la trayectoria deseada. Ahora bien, el problema de diseño (síntesis) debe de establecerse como un problema de optimización numérica [9], en donde la función objetivo, las variables de diseño y las restricciones son detalladas seguidamente.

\section{Función objetivo}

Se establece como función objetivo $I_{e}$ (ecuación 13), la integral de la raíz cuadrada del cuadrado del error de las $n$ coordenadas de la trayectoria deseada $\left(X_{D}, Y_{D}\right)$ y las $\mathrm{n}$ coordenadas generadas por el M4B $\left(X_{\text {sis }}, Y_{\text {sis }}\right)$ en el punto $\left(P_{x}, P_{y}\right)$, donde $\mathrm{n}=12$.

$$
I_{e}=\int_{i=1}^{n=12} \sqrt{\left(X_{D}-X_{S i s}\right)^{2}+\left(Y_{D}-Y_{s i s}\right)^{2}}
$$

\section{Vector de variable de diseño}

Se establecen 4 vectores de diseño $v$ en donde el subíndice representa el número de caso al que corresponde cada vector (ecuaciones 14-17).

$v_{1}=\left[r_{1}, r_{2}, r_{3}, r_{4}\right]$

$v_{2}=\left[r_{1}, r_{2}, r_{3}, r_{4}, \theta_{1}\right]$

$v_{3}=\left[r_{1}, r_{2}, r_{3}, r_{4}, \theta_{1}, l\right]$

$v_{4}=\left[r_{1}, r_{2}, r_{3}, r_{4}, \theta_{1}, l, \alpha\right]$

\section{Restricciones}

Se establece como única restricción el criterio de Grashof para mecanismos de cuatro eslabones [10], éste establece que, si la suma de las longitudes del eslabón más corto y largo es menor o igual a la suma de los dos eslabones restantes, entonces al menos un eslabón puede rotar completamente. Esta restricción se muestra en la ecuación 18.

$l+s \leq p+q$
Donde $l$ es el eslabón más largo, $s$ el más corto, $p$ y $q$ los eslabones acoplador y oscilador respectivamente.

\section{Problema de optimización general}

Un problema de optimización general es [9]:

$\min$ o $\max f(x)$

Sujeto a:

$g(x) \leq 0 \quad \in \mathbb{R}^{m}$

$h(x)=0 \quad \in \mathbb{R}^{k}$

$x_{\min }<x<x_{\max } \in \mathbb{R}^{p}$

donde:

$x$ es un vector de diseño n-dimensional.

$f(x)$ es la función objetivo.

$g(x)$ son las restricciones de desigualdad.

$h(x)$ son las restricciones de igualdad.

\section{Problema de optimización del M4B}

Sea el problema de optimización numérica descrito por la ecuación 23, donde se minimiza la función objetivo (ecuación 13), para obtener la solución al problema de síntesis dimensional para el seguimiento de trayectoria de un M4B.

$$
\begin{aligned}
& \min f\left(v_{j}\right)=\int_{i=1}^{n=1} \sqrt{\left(X_{D}-X_{S i s}\right)^{2}+\left(Y_{D}-Y_{\text {sis }}\right)^{2}} \\
& v_{j} \in \mathbb{R}^{q} \quad \begin{array}{l}
\forall j=1, \ldots, 4 \\
\forall q=4, \cdots, 7
\end{array}
\end{aligned}
$$

Sujeto a:

$g_{j}\left(v_{j}\right)=r_{1}+r_{2}-r_{3}-r_{4} \leq 0 \quad \forall j=1, \ldots, 4$

Con

$v_{j_{\text {min }}}<v_{j}<v_{j_{\max }} \quad \forall j=1, \ldots, 4$

\begin{tabular}{|l|r|r|r|r|r|r|r|}
\hline & $\boldsymbol{r}_{\mathbf{1}}$ & $\boldsymbol{r}_{\mathbf{2}}$ & $\boldsymbol{r}_{\mathbf{3}}$ & $\boldsymbol{r}_{\mathbf{4}}$ & $\boldsymbol{T}_{\mathbf{1}}$ & \multicolumn{1}{c|}{$\boldsymbol{\alpha}$} & $\boldsymbol{\alpha}$ \\
\hline$v_{1_{\max }}$ & 250 & 250 & 250 & 250 & - & - & - \\
\hline$v_{1_{\min }}$ & 5 & 5 & 5 & 5 & - & - & - \\
\hline$v_{2_{\max }}$ & 250 & 250 & 250 & 250 & $2 \pi$ & - & - \\
\hline$v_{2_{\min }}$ & 5 & 5 & 5 & 5 & 0 & - & - \\
\hline$v_{3_{\max }}$ & 250 & 250 & 250 & 250 & $2 \pi$ & 50 & - \\
\hline$v_{3_{\min }}$ & 5 & 5 & 5 & 5 & 0 & 5 & - \\
\hline$v_{4_{\max }}$ & 250 & 250 & 250 & 250 & $2 \pi$ & 50 & 2.3562 \\
\hline$v_{4_{\min }}$ & 5 & 5 & 5 & 5 & 0 & 5 & 0.7854 \\
\hline
\end{tabular}

Tabla 2 Límites de las variables de diseño

FOSTER-VÁZQUEZ, Ixshel Jhoselyn, PORTILLO-VÉLEZ, Rogelio de Jesús y VAZQUEZ-SANTACRUZ, Eduardo. Sobre la síntesis óptima de mecanismos. Revista de Simulación y Laboratorio. 2019. 


\section{Metodología}

El proceso para resolver un problema de síntesis no siempre es el mismo, éste puede variar desde el tipo de mecanismo, configuración, tarea por realizar, entre otras cosas, sin embargo, la importancia de cómo se plantea cada problema recae fuertemente en la obtención de la solución. Los pasos que se realizaron para resolver el problema de síntesis se detallan seguidamente:

1. Determinar el tipo de síntesis a realizar como se describe en la sección 2. En el caso de la síntesis dimensional se debe predefinir el mecanismo y la configuración que éste tendrá (caso del M4B).

2. Seleccionar la tarea que realizará el mecanismo, para el caso específico de este trabajo se desea realizar un seguimiento de trayectoria.

3. Obtener la cinemática del mecanismo y la cinemática del punto que seguirá la trayectoria deseada (acoplador).

4. Definir la trayectoria deseada.

5. Establecer la estrategia de optimización, para este trabajo se definió el problema de síntesis como un problema de optimización numérica.

6. Definir las partes matemáticas que conforman la estrategia de optimización:
a. Función objetivo.
b. Vector de variables de diseño.
c. Restricciones.

7. Definir el problema de optimización

8. Regresar a alguno de los pasos anteriores si es necesario.

Los pasos mencionados anteriormente, son una explicación de cómo se fue planteando el problema de síntesis. El punto 6 inciso b, fue un proceso que se realizó un total de 4 veces y es una parte importante para la obtención de los resultados, se presenta en la figura 3 el diagrama de flujo de los pasos presentados anteriormente.
Junio 2019 Vol.6 No.19 16-22

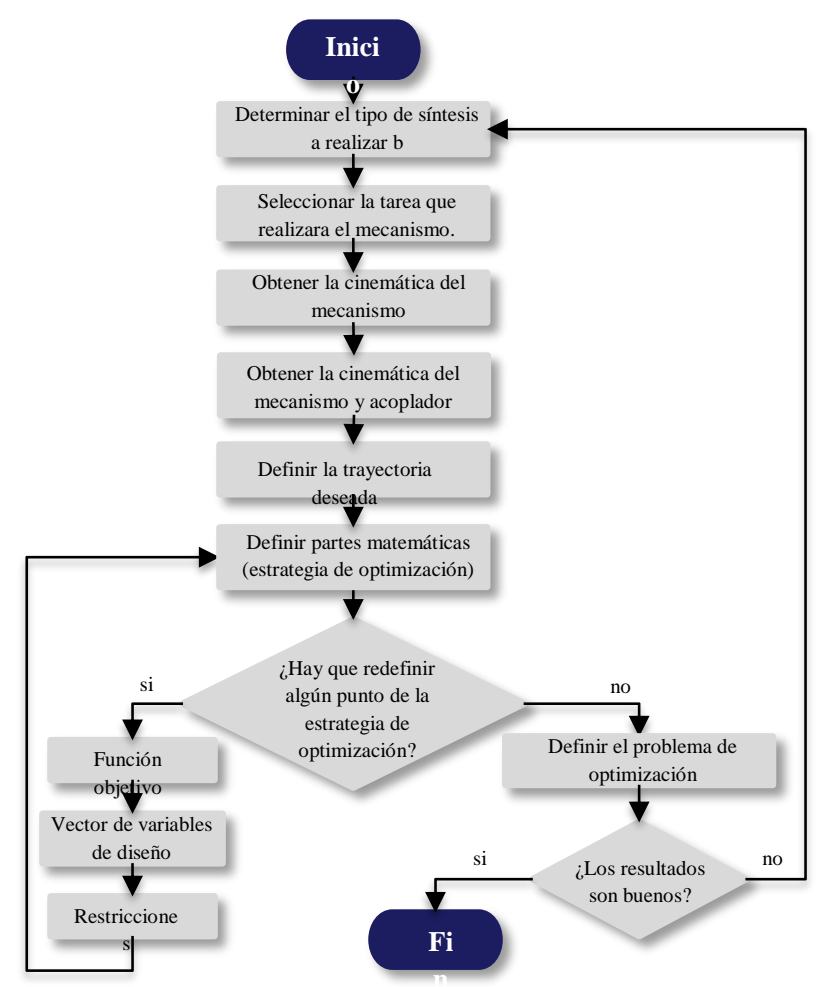

Figura 1 Diagrama de flujo de la metodología desarrollada

Después de definir la metodología, se procedió a la escritura del leguaje computacional utilizado como herramienta de trabajo el Toolbox de algoritmos genéticos de Matlab®.

\section{Resultados}

Se realizó un total de 10 ejecuciones por caso mostrando en cada una de ellas una tabla con los valores obtenidos para el vector de diseño en cada ejecución, señalando con rojo el mejor resultado, de igual forma se calcula la media del índice del error y se muestra el gráfico de la mejor ejecución con un acercamiento de la trayectoria obtenida por el mecanismo (puntos negros) y la trayectoria deseada (puntos magenta).

\section{Caso 1}

Para el caso 1, se muestra la tabla 3 con los resultados obtenidos para el vector de diseño de la ecuación 14, la mejor corrida está resaltada en color rojo, seguidamente se muestra en el grafico 1 la simulación del mejor resultado obtenido en el proceso de optimización, junto con un acercamiento de la trayectoria obtenida del mecanismo y la trayectoria deseada. 


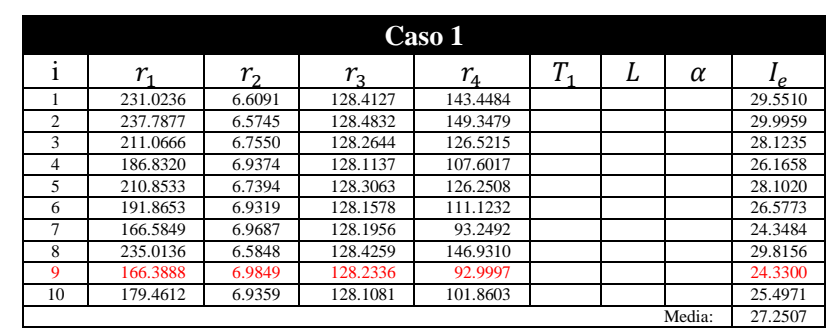

Tabla 3 Resultados obtenidos (caso 1)

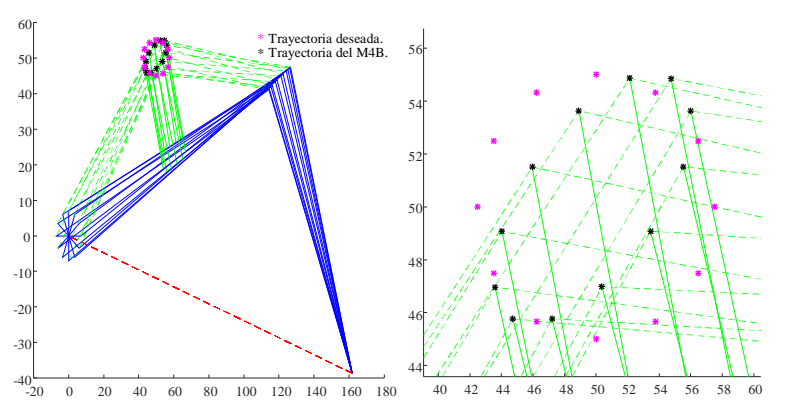

Gráfico 1 Mejor simulación del caso 1

\section{Caso 2}

Para el caso 2, se muestra la tabla 4 con los resultados obtenidos para el vector de diseño de la ecuación 15, la mejor corrida se indica con el color rojo, de igual forma se muestra en el grafico 2 la simulación del mejor resultado obtenido en el proceso de optimización, junto con un acercamiento de la trayectoria obtenida del mecanismo y la trayectoria deseada.

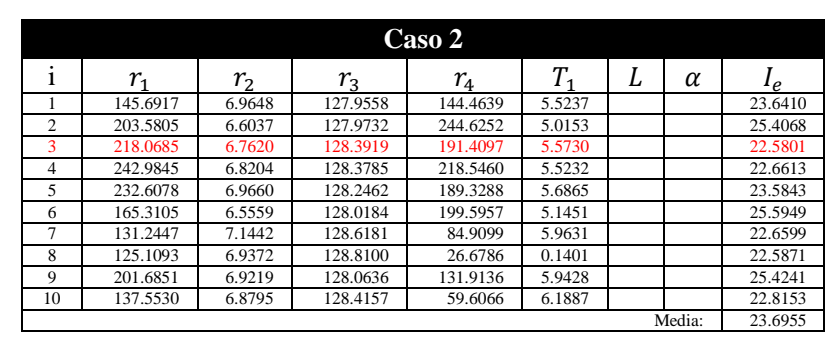

Tabla 4 Resultados obtenidos (caso 2)

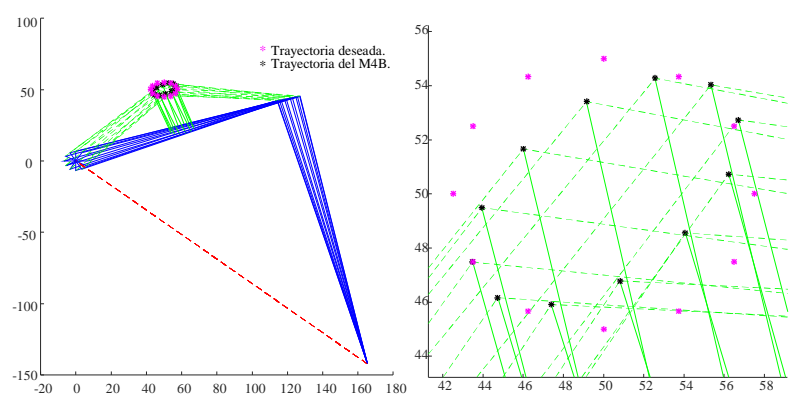

Gráfico 2 Mejor simulación del caso 2

\section{Caso 3}

Para el caso 3, se muestra la tabla 5 con los resultados obtenidos para el vector de diseño de la ecuación 16 , se resalta con rojo el resultado de la mejor corrida, seguidamente se muestra en el grafico 3 la simulación del mejor resultado obtenido en el proceso de optimización, un acercamiento de la trayectoria obtenida del mecanismo y la trayectoria deseada.

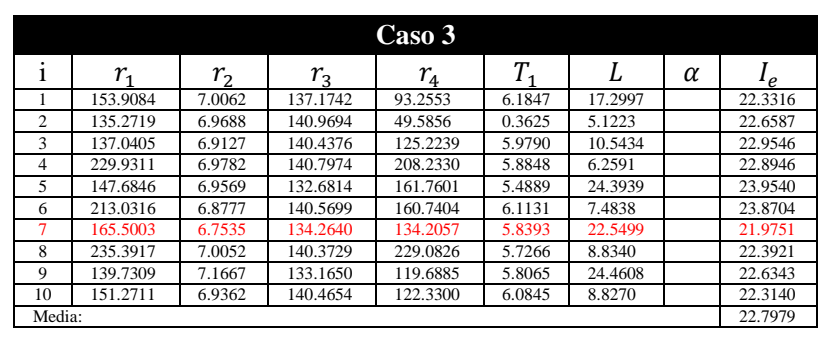

Tabla 5 Resultados obtenidos (caso 3)

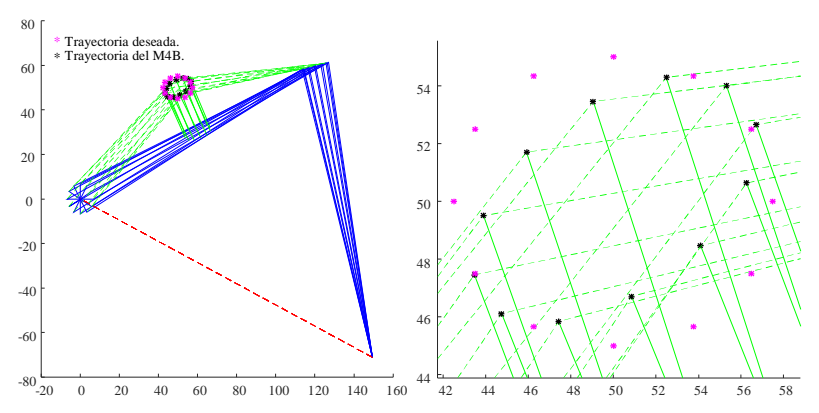

Gráfico 3 Mejor simulación del caso

\section{Caso 4}

Por último, para el caso 4 se muestra en la tabla 6 los resultados obtenidos para el vector de diseño de la ecuación 17, se resalta con rojo la mejor corrida, se muestra en el grafico 4 la simulación del mejor resultado obtenido en el proceso de optimización, junto con un acercamiento de la trayectoria obtenida del mecanismo y la trayectoria deseada. Cabe resaltar que este último es el mejor resultado obtenido de todas las simulaciones realizadas en el proceso de optimización.

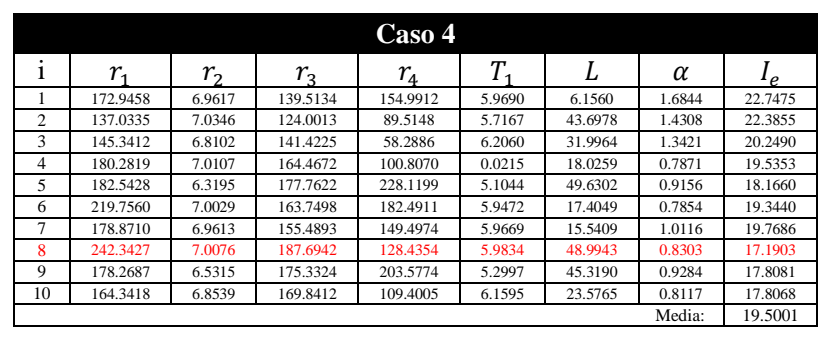

Tabla 6 Resultados obtenidos (caso 4) 


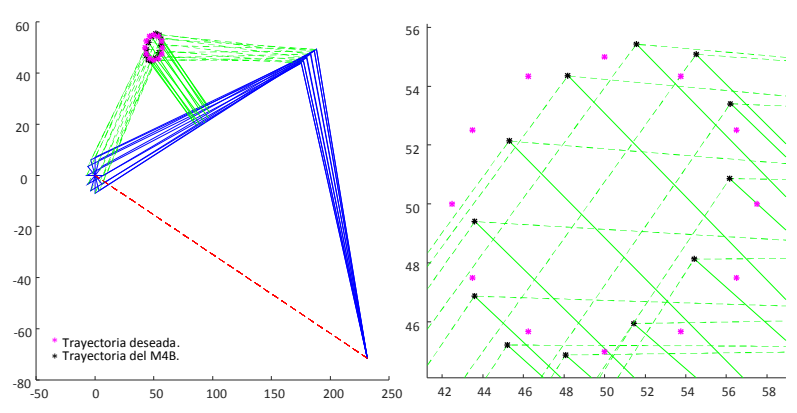

Gráfico 4 Mejor simulación del caso 4

\section{Conclusiones}

En este trabajo se presentan 4 casos, en cada uno de ellos se plantea un vector de diseño diferente o simplemente modificado. En los resultados se observa que gracias a la modificación del vector de diseño se va obteniendo un mejor resultado y éste se puede comprobar con el valor de la media siendo el caso 4 ejecución 8 el mejor resultado obtenido en este trabajo.

La importancia de un buen planteamiento influye en la obtención de resultados satisfactorios y puede reducir el tiempo de búsqueda de una o un grupo de soluciones, el presente trabajo comenzó con la necesidad de plantear un problema de optimización de síntesis dimensional utilizado como técnica numérica algoritmos genéticos.

La importancia de los resultados mostrados en este trabajo es la disminución del índice de error en función del cambio realizado en el vector de diseño y se considera que la contribución de este artículo esta situada en un contexto ingenieril y académico.

\section{Referencias}

[1] Vallejo, J., Avil, R., Hernández, A., \& Amezua, E. (1995). Nonlinear optimization of planar linkages for kinematic syntheses. Mechanism and Machine Theory, 30(4), 501-518.

[2] Vasiliu, A., \& Yannou, B. (2001). Dimensional synthesis of planar mechanisms using neural networks: application to path generator linkages. Mechanism and Machine Theory, 36(2), 299-310.
[3] Quintero, H. F., TRUJILLO, G. C., \& Arias, A. D. (2004). Síntesis de generación de trayectoria y de movimiento para múltiples posiciones en mecanismos, utilizando algoritmos genéticos. Scientia et technica, 10(25), 131-136.

[4] Cabrera, J. A., Simon, A., \& Prado, M. (2002). Optimal synthesis of mechanisms with genetic algorithms. Mechanism and machine theory, 37(10), 1165-1177.

[5] Vega-Alvarado, E., Santiago-Valentín, E., Sánchez-Márquez, A., Solano-Palma, A., Portilla-Flores, E. A., \& Flores-Pulido, L. (2014). Síntesis óptima de un mecanismo plano para seguimiento de trayectoria utilizando evolución diferencial. Research in Computing Science, 72, 85-98.

[6] Shigley, J. E., Uicker, J. J., Pérez, J. H., \& de Contín, H. C. (1983). Teoría de máquinas y mecanismos (No. TJ145. S54 1983.). México; McGraw-Hill.

[7] Moreno, R. P. (2006). Análisis de mecanismos y problemas resueltos. Alfaomega.

[8] Goldberg, David E., Genetic Algorithms in Search, Optimization \& Machine Learning, Addison-Wesley, 1989.

[9] Chapra, S. C., \& Canale, R. P. (2007). Métodos numéricos para ingenieros. McGraw-Hill.

[10] Grashof, F. (1875). Theoretische Maschinenlehre. 1 (Vol. 2). Voss.

[11] Norton, R. L. (1995). Diseño de maquinaria: Una introducción a la síntesis y al análisis de mecanismos y maquinas (No.TJ230. N677 1994.). 